\title{
Invasive pulmonary aspergillosis in non-immunocompromised patients
}

\author{
Jonathan L Hovenden, Frank Nicklason, Rosemary A Barnes
}

Invasive pulmonary aspergillosis can occur in non-immunocompromised patients: consider diagnostic bronchoscopy or open lung biopsy in any patient with progressive pulmonary infiltrates to conventional antimicrobial treatment. who fails to respond

Profound neutropenia is the most common predisposing feature in invasive aspergillosis. ${ }^{1}$ Classically, patients receiving cytotoxic chemotherapy for acute haematological malignancy, recipients of organ transplants, and those on high doses of systemic corticosteroids ${ }^{2}$ are most at risk. Chronic granulomatous disease, alcoholism, influenza, diabetes, and parenteral antibiotics have also occasionally been implicated as predisposing factors. ${ }^{3.6}$ Invasive aspergillosis is very rare outside these groups. We report on two patients with fatal pulmonary aspergillosis in whom early suspicion and invasive diagnostic procedures may have affected outcome.

\section{Case 1}

An 81 year old retired coal miner with chronic obstructive airways disease presented with a two day history of left sided pleuritic chest pain, fever, and breathlessness at rest. He complained of increased coughing. He smoked 20 cigarettes a day but denied alcohol abuse. There was no history of systemic corticosteroid use, diabetes, malignant disease, or immunological disorder. He had been admitted seven weeks previously for cholangitis, which settled on antibiotic treatment. He took terbutaline by metered aerosol and $400 \mu \mathrm{g}$ budesonide (a corticosteroid) four times a day by inhaler. Oral amoxycillin was started 24 hours before admission.

On admission he had fever and tachypnoea and was expectorating green sputum. Auscultation showed bilateral coarse crepitations but no evidence of consolidation. He had a white cell count of $13 \cdot 1 \times 10^{4} / 1$ (neutrophil count $10.0 \times 10^{4} / \mathrm{l}$ ) and hypoxia. Intravenous cefuroxime and oral prednisolone at $10 \mathrm{mg}$ four times a day were started, and his fever settled. A ventilation-perfusion scan performed on the 3rd day showed normal ventilation but bilateral perfusion defects. Anticoagulation with heparin was started for suspected pulmonary embolism. Two days later fever and pleuritic pain recurred. A chest radiograph showed progressive left upper lobe consolidation. Blood cultures were sterile, and sputum cultures

\footnotetext{
Department of Medical Microbiology, University of Wales College of Medicine, Cardiff CF4 4XN

Jonathan L Hovenden, MB, registrar

Rosemary A Barnes, MI, lecturer
}

Department of Geriatric Medicine, University of Wales College of Medicine, Cardiff CF4 4XN

Frank Nicklason, MB, senior registrar

Correspondence to: Dr Hovenden.

$B M 71991 ; 302: 583-4$

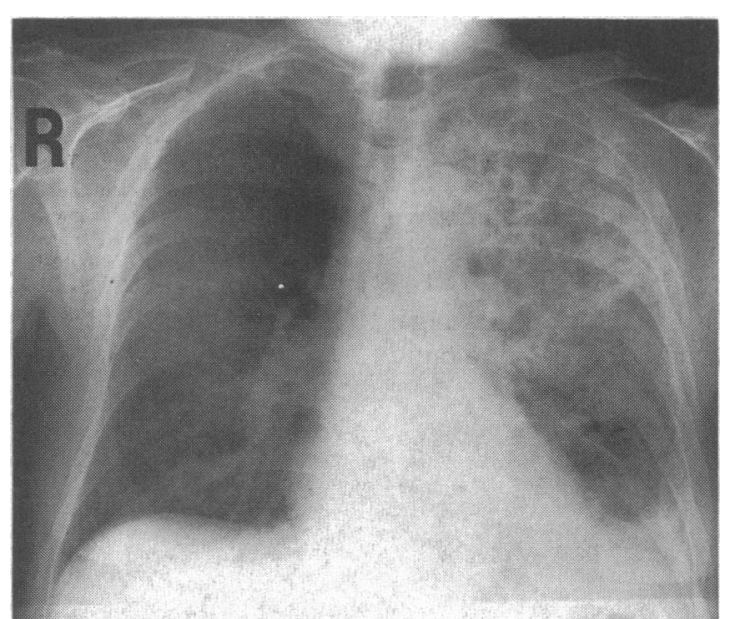

Chest radiograph of patient in case one on 24th day in hospital showed normal flora. Aspergillus was cultured from sputum on the 13th hospital day but was not thought to be clinically important. We changed the antibiotics to ciprofloxacin, erythromycin, gentamicin, and metronidazole. Hypoxaemia, conscious level, and renal function deteriorated, and the patient died three days later. Antibody titres to Legionella pneumophila, Mycoplasma pneumoniae, Chlamydia psittaci, and influenza A and B viruses were not raised.

Necropsy showed organised thrombus in the major arteries supplying upper and lower lobes bilaterally. There was a well demarcated area of purulent cavitation and parenchymal destruction in the left upper lobe. Histological examination of this area showed hyphal invasion of blood vessels characteristic of invasive aspergillosis, and culture yielded Aspergillus fumigatus. There was no evidence of aspergillosis in any other organ.

\section{Case 2}

A 61 year old man was admitted with a three day history of agitation, confusion, and dehydration after a flu-like illness 10 days previously. He had a history of peptic ulceration, had smoked 60 cigarettes a day for the past 40 years, and had a moderate alcohol intake. He had attended a nearby hospital five months previously as an outpatient for a urological opinion (recurrent urinary tract infection). He had no fever but was extremely agitated and disorientated. There was reduced air entry into his chest with crepitations at both bases. Otherwise, examination gave normal results. His white cell count was $24 \times 10^{\%} / 1$ with a considerable neutrophilia, and measurement of blood gases showed hypoxia (oxygen pressure $7.73 \mathrm{kPa}$ ). A chest radiograph showed widespread patchy consolidation suggesting bronchopneumonia. He was unable to expectorate any sputum. We considered a diagnosis of a toxic confusional state secondary to acute pneumonia, and he was started on $1 \mathrm{~g}$ erythromycin four times a day and $750 \mathrm{mg}$ cefuroxime three times a day intravenously with intravenous fluid replacement. The next day computed tomography without contrast showed mild cerebral atrophy only (the procedure was limited by the patient moving) and lumbar puncture gave normal results. He continued to deteriorate and developed a fever. The dose of cefuroxime was increased to $1.5 \mathrm{~g}$ three times a day. Tests for legionella antibodies gave negative results but viral tests showed a titre of $1 / 256$ to influenza A virus suggesting recent infection. Gentamicin was added but his condition worsened and an acute respiratory distress syndrome developed. Four days after admission he was transferred to the intensive care unit for assisted ventilation and inotropic support. High dosages of cotrimoxazole $(120 \mathrm{mg} / \mathrm{kg} /$ day $)$ and acyclovir were started intravenously. Sputum taken at this stage by tracheal aspiration grew a heavy, pure growth of $A$ fumigatus. Amphotericin at $1 \mathrm{mg} / \mathrm{kg} /$ day was started, but he developed multiorgan failure and died three days later. Necropsy showed a diffuse necrotising pneumonitis with numerous microabscesses associated with branching septate hyphae typical of invasive aspergil- 
losis. There was no evidence of aspergillosis in brain, liver, spleen, kidney, or thyroid.

\section{Discussion}

These patients had none of the classic predisposing factors for invasive aspergillosis. Although systemic steroids were used briefly in the first patient, and though corticosteroids enhance tissue damage and invasion, ${ }^{2}$ it is unlikely that they predisposed to the disease, though they may have influenced the outcome. The role of inhaled steroids in these circumstances has not been discussed but they have been shown to increase the incidence of superficial fungal infection and may have predisposed to invasive disease in the first patient.

Alcohol abuse is known to be a risk factor ${ }^{4}$ presumably by affecting the function of neutrophils ${ }^{7}$ and macrophages. ${ }^{8}$ There are few published case reports of invasive pulmonary aspergillosis after influenza in patients not receiving immunosuppressive treatment. ${ }^{59}$ The mechanism by which influenza predisposes to invasive pulmonary aspergillosis is unknown but may involve suppression of cell mediated immunity. ${ }^{10}$ In one review of 25 definite and seven possible cases in non-immunocompromised patients four had evidence of recent influenza A infection. ${ }^{4}$ We believe that influenza may have predisposed to aspergillus invasion in the second patient.

The role of prolonged use of antibiotics in predisposing to invasive aspergillosis is less clear. Most clinical studies have not shown that antibiotic treatment is more common in those with disseminated aspergillosis than similarly immunocompromised patients. The persistence of fever during antibiotic treatment may be a more reliable index of fungal disease than is duration of antibiotic treatment. ${ }^{.1}$

An association between building work and outbreaks of invasive aspergillosis is recognised. ${ }^{12}$ At the time that the second patient developed aspergillosis major building work was in progress near the intensive care unit, and there had been an increased isolation rate of aspergillus from sputum in some inpatients without evidence of infection. Environmental sampling had not shown contamination of areas of patient care with fungal spores. The second patient had been in the hospital for only four days before transfer to the unit with established respiratory failure, and he died shortly after; nosocomial infection was therefore thought to be unlikely. The first patient was nursed in a different hospital, and there was no known association with building work.

Isolating filamentous fungi in sputum from nonneutropenic patients is usually thought to reflect colonisation and not invasion. This may lead to a delay in diagnosis and the start of antifungal treatment. In a retrospective analysis of aspergillus isolates from the sputum of 10 non-immunocompromised patients with progressive pulmonary infiltrates, two had invasive pulmonary aspergillosis. ${ }^{+}$Chronic lung disease and cigarette smoking have, however, been associated with high colonisation rates, ${ }^{13}$ so the presence of aspergillus in the sputum should not be used to diagnose invasive. pulmonary aspergillosis but should arouse suspicion in a patient with progressive lung infiltrates.

One study has suggested that detecting fungal elements in bronchoalveolar lavage fluid can be a rapid and effective technique for diagnosing invasive pulmonary aspergillosis in immunocompromised patients with new pulmonary infiltrates. ${ }^{14}$ This technique, however, may not distinguish colonisation from invasion in non-immunocompromised patients. There is much controversy about using transbronchial and open lung biopsy in managing patients with pulmonary infiltrates. Biopsy may yield a diagnosis and lead to a change in treatment in up to $45 \%$ of patients, ${ }^{15}$ but this may not influence clinical outcome. ${ }^{15} 16$ These findings cannot be extrapolated to non-immunosuppressed patients because of differences in underlying disease. In most studies of invasive diagnostic procedures complication rates and mortality have been low. ${ }^{17}$

Detecting aspergillus antigen in serum and urine by enzyme linked immunosorbent assay (ELISA) has recently been shown to predict the onset of invasive pulmonary aspergillosis in profoundly neutropenic patients after treatment for leukaemia and bone marrow transplantation with positive and negative predictive values of greater than $95 \% .{ }^{18}$ At present these assays are not widely available and there is currently little data on their use in immunocompetent patients, in whom efficient antibody responses are likely to result in rapid clearing of antigenaemia.

We believe that the use of more invasive procedures may have led to treatment being started earlier in both patients. Bronchoalveolar lavage and transbronchial biopsy or open lung biopsy should be considered early in certain patients with progressive pulmonary infiltrates not responding to empirical treatment.

We thank Professor P Harper and Professor M Pathy for permission to study these case histories.

1 Gerson SL, Talbot GH, Hurwitz S, Strom S, Lusk EJ, Cassileth PA Prolonged granulocytopenia: the major risk factor for invasive pulmonary aspergillosis in patients with acute leukemia. Ann Intern Med 1984;100: $345-51$.

2 Rinaldi MG. Invasive aspergillosis. Rev Infect Dis 1983;5:1061-77.

3 Lazrus GH, Neu HM. Agents responsible for infection in chronic granulomatous disease of childhood. 7 Pediatr 1975;86:415-7.

$4 \mathrm{Karam} \mathrm{GH}$, Griffin FM. Invasive pulmonary aspergillosis in non-immunocompromised, non-neutropenic hosts. Rev Infect Dis 1986;3:357-63.

5 Horn CR, Wood NC, Hughes JA. Invasive aspergillosis following postinfluenzal pneumonia. British fournal of Diseases of the Chest 1983;77: influenz: $407-10$.

6 D'Silva H, Burke JF, Cho SY. Disseminated aspergillosis in an apparently immunocompetent host. FAMA 1982;248:1495-7.

7 Ascah KJ, Hyland RH, Hutcheon MA, et al. Invasive aspergillosis in "healthy" patient. Can Med Assoc f 1984;131:332-5.

8 Louria DB, Almy TP. Susceptibility to infection during experimental alcoho intoxication. Trans Assoc Am Physicians 1963;76:102-12.

9 Fischer JJ, Walker DH. Invasive pulmonary aspergillosis associated with influenza. FAMA 1979;241:1493-4.

10 Scheinberg MA, Blacklow NR, Goldstein AL, Parrino TA, Rose FB, Cathcar ES. Influenza: response of T-cell lymphopenia to thymosin. $N$ Engl f Med 1976;294:1208-11.

11 Gerson SL, Talbot GH, Lusk E, Hurwitz S, Strom BL, Cassileth PA. Invasive pulmonary aspergillosis in adult leukemia: clinical clues to its diagnosis. 7 Clin Oncol 1985;3:1109-16.

12 Barnes RA, Rogers TRF. Control of an outbreak of nosocomial aspergillosis by laminar air-flow isolation. F Hosp Inf 1989;14:89-94.

$13 \mathrm{Yu} \mathrm{L}$, Muder RR, Poorsattar A. Significance of isolation of aspergillus from the respiratory tract in diagnosis of invasive pulmonary aspergillosis. Results from a three-year prospective study. Am 7 Med 1986;81:249-54.

$14 \mathrm{Kahn} \mathrm{FW,} \mathrm{Jones} \mathrm{JM,} \mathrm{England} \mathrm{DM.} \mathrm{The} \mathrm{role} \mathrm{of} \mathrm{bronchoalveolar} \mathrm{lavage} \mathrm{in} \mathrm{the}$ diagnosis of invasive pulmonary aspergillosis. Am f Clin Pathol 1986;86 518-23.

15 Rossiter SJ, Miller CM, Churg AM, Carrington CB, Mark JBD. Open lung biopsy in the immunosuppressed patient. Is it really beneficial? $\mathcal{F}$ Thorac Cardiovasc Surg 1979;77:338-45.

16 McCabe RE, Brooks RG, Mark JBD, Remington JS. Open lung biopsy in patients with acute leukemia. Am J Med 1985;78:609-16.

17 Bartlett JG. Invasive diagnostic techniques in pulmonary infections. In Pennington JE, ed. Respiratory infections: diagnosis and management. 2nd ed. New York: Raven Press, 1988:69-96.

18 Rogers TR, Haynes KA, Barnes RA. Value of antigen detection in predicting invasive pulmonary aspergillosis. Lancet 1990;336:1210-3.

(Accepted 10 fanuary 1991) 